\title{
Constructing 2-Arc-Transitive Covers of Hypercubes
}

\author{
Michael Giudici ${ }^{1 *}$, Cai Heng $\mathrm{Li}^{2 \dagger}$, Yian $\mathrm{Xu}^{3 \ddagger}$ \\ ${ }^{1}$ Centre for the Mathematics of Symmetry and Computation, \\ School of Mathematics and Statistics, \\ The University of Western Australia \\ ${ }^{2}$ Department of Mathematics \\ Southern University of Science and Technology \\ 3 School of Mathematics \\ Southeast University
}

\begin{abstract}
We introduce the notion of a symmetric basis of a vector space equipped with a quadratic form, and provide a sufficient and necessary condition for the existence to such a basis. Symmetric bases are then used to study Cayley graphs of certain extraspecial 2-groups of order $2^{2 r+1}(r \geq 1)$, which are further shown to be normal Cayley graphs and 2-arc-transitive covers of $2 r$-dimensional hypercubes.

Keywords extraspecial 2-group symmetric basis quadratic form locally-primitive graph normal Cayley graph
\end{abstract}

\section{Introduction}

Throughout this paper, all graphs are simple, connected and regular. Let $\Gamma$ be a graph with vertex set $V(\Gamma)$ and edge set $E(\Gamma)$. An $s$-arc of $\Gamma$ is a sequence $\left(v_{1}, \ldots, v_{s+1}\right)$ of $s+1$ vertices such that for all $1 \leq i \leq s,\left\{v_{i}, v_{i+1}\right\}$ is an edge in $\Gamma$ and $v_{i} \neq v_{i+2}$, and $\Gamma$ is said to be $s$-arc-transitive if the automorphism group of $\Gamma$ is transitive on the set of $s$-arcs. The study of $s$-arc-transitive graphs is motivated by a result of Tutte (1949), which says that there are no $s$-arc-transitive graphs of valency 3 for $s \geq 6$. Later this result was extended by Weiss [16] saying that there are no 8-arc-transitive graphs of valency at least 3. Thus analysing the $s$-arc-transitive graphs for $2 \leq s \leq 7$ has become one of the central goals in algebraic graph theory, and the classification of some 2-arc-transitive graphs has been obtained. For example, the 2-arc-transitive circulants are classified in [1]; a complete classification of 2-arc-transitive dihedrants is given in [7]; and a class of 2-arc-transitive Cayley graphs of elementary abelian 2-groups is classified in [9].

A natural idea to investigate the 2 -arc-transitive graphs is to study their quotient graphs. Let $\mathcal{P}$ be a partition of the vertex set $V(\Gamma)$. Define the quotient graph $\Gamma_{\mathcal{P}}$ of $\Gamma$ to be the graph with vertex set $\mathcal{P}$ and two parts $P, P^{\prime} \in \mathcal{P}$ form an edge if and only if there is at least one edge in $\Gamma$ joining a vertex of $P$

\footnotetext{
*Email: michael.giudici@research.uwa.edu.au.

${ }^{\dagger}$ Email: lich@sustc.edu.cn.

${ }^{\ddagger}$ Email: yian.xu@research.uwa.edu.au.
} 
and a vertex of $P^{\prime}$. If $\mathcal{P}$ is $G$-invariant for some group $G$ of automorphisms of $\Gamma$, then the action of $G$ on $\Gamma$ induces an action of $G$ on $\Gamma_{\mathcal{P}}$. Let $N$ be a nontrivial normal subgroup of $G$ and $\mathcal{P}$ be the set of $N$-orbits in $V(\Gamma)$. The quotient graph $\Gamma_{\mathcal{P}}$ is said to be a normal quotient of $\Gamma$, denoted $\Gamma_{N}$. In general, the valency of $\Gamma_{N}$ divides the valency of $\Gamma$. If the valency of $\Gamma$ equals the valency of $\Gamma_{N}$, then $\Gamma$ is said to be a cover of $\Gamma_{N}$. It has been proved by Praeger [11, Theorem 4.1] that if $G$ is vertex-transitive and 2-arc-transitive on $\Gamma$, and $N$ has more than two orbits in $V(\Gamma)$, then

1. $G / N$ is $s$-arc transitive on $\Gamma_{N}$ and $G / N$ is faithful on $V\left(\Gamma_{N}\right)$,

2. $\Gamma$ is a cover of $\Gamma_{N}$, and

3. $N$ is semiregular on $V(\Gamma)$.

We say that a permutation group is quasiprimitive on a set $\Omega$ if every nontrivial normal subgroup of the permutation group is transitive on $\Omega$, and primitive if it acts transitively on $\Omega$ and preserves no nontrivial partition of $\Omega$. A permutation group is said to be bi-quasiprimitive on $\Omega$ if

(i) each nontrivial normal subgroup of the permutation group has at most two orbits on $\Omega$, and

(ii) there exists a normal subgroup with two orbits on $\Omega$.

The structure of finite quasiprimitive permutation groups was investigated in [11 and the types of quasiprimitive groups that are 2-arc-transitive on a graph were determined. Praeger studied biquasiprimitive groups in [12] and one specific class identified was previously studied in [10]. One family of such bipartite bi-quasiprimitive graphs are the affine ones. A 2-arc-transitive graph is said to be affine, if there is a vector space $N$ and a group $G$ of automorphisms of the graph such that $N \leq G \leq A G L(N)$ with $N$ regular on the vertices and $G$ acting transitively on the set of 2 -arcs. Table 1 in [9] classifies all affine bipartite 2-arc-transitive graphs with the stabilizer of the bipartition of the vertices being primitive on each bipartition.

Another interesting topic is to reconstruct 2-arc-transitive covers of 2-arc-transitive graphs. It is known that every finite regular graph has a 2-arc-transitive cover [2]. In [8] Du, Malnič and Waller investigate the regular covers of complete graphs which are 2-arc-transitive, and they give a complete classification of all graphs whose group of covering transformations is either cyclic or isomorphic to $Z_{p} \times Z_{p}$ where $p$ is a prime and whose fibre-preserving subgroup of automorphisms acts 2-arc-transitively. In particular, two families of 2-arc-transitive graphs are obtained. After that, many more results related to the reconstruction of the 2-arc-transitive graphs have been obtained, see [6] for examples.

The main subject of this paper is to construct a 2-arc-transitive cover for one family of affine graphs, namely the hypercubes. Let $V=\mathbb{Z}_{2}^{d}$ be a $d$-dimensional vector space over the field $\mathbb{F}_{2}$, let $e_{1}, \ldots, e_{d}$ be a basis of $V$ and $\mathcal{B}=\left\{e_{1}, \ldots, e_{d}\right\}$. A $d$-dimensional hypercube is a Cayley graph $Q_{d}=\operatorname{Cay}(V, \mathcal{B})$. It is known that $Q_{d}$ admits a regular group of automorphisms $Z_{2}^{d}$ and $A u t\left(Q_{d}\right)=Z_{2}^{d} \rtimes S_{d}$, where $S_{d}=\operatorname{Aut}_{\mathbf{1}}\left(Q_{d}\right)$ and permutes $e_{1}, \ldots, e_{d}$ naturally (see [15]). Thus the hypercubes are 2-arc-transitive affine graphs. Furthermore, it has been shown in [9] that $Q_{d}$ is bi-quasiprimitive if and only if $d=2$ or $d$ is odd. In this paper, we are interested in the even-dimensional hypercubes, in particular, we construct a 2-arc-transitive cover for even-dimensional hypercubes. We also show that such a cover is a normal Cayley graph.

Let $G$ be a finite group, and $S$ be a subset of $G$ such that $S$ does not contain the identity of $G$ and $S=S^{-1}=\left\{s^{-1} \mid s \in S\right\}$. We say that an element $g$ of $G$ is an involution if it has order 2 , that 
is, $g^{2}=\mathbf{1}$ and $g \neq \mathbf{1}$. The Cayley $\operatorname{graph} \Gamma=\operatorname{Cay}(G, S)$ is defined to have vertex set $V(\Gamma)=G$, and edge set $E(\Gamma)=\{\{g, s g\} \mid s \in S\}$. It is well known that a graph is a Cayley graph if and only if its full automorphism group contains a subgroup acting regularly on the vertex set of the graph (see [14]).

Let $\Gamma=\operatorname{Cay}(G, S)$ be a Cayley graph for some group $G$ and $A u t(\Gamma)$ be the full automorphism group of $\Gamma$. For each $g \in G$, define a map $\hat{g}: G \rightarrow G$ by the right multiplication of $g$ on $G$ as below:

$$
\hat{g}: x \rightarrow x g, \text { for } x \in G \text {. }
$$

Then $\hat{g}$ is an automorphism of $\Gamma$. It follows from the definition that the group $\hat{G}=\{\hat{g} \mid g \in G\}$ is a subgroup of $\operatorname{Aut}(\Gamma)$ and acts regularly on $V(\Gamma)$. Following Xu [18, we say that $\Gamma$ is a normal Cayley graph for $G$ (or normal) if $\hat{G} \unlhd A u t(\Gamma)$, otherwise we say that $\Gamma$ is a non-normal Cayley graph for $G$ (or non-normal).

Suppose that $V$ is a $d$-dimensional vector space with a nondegenerate quadratic form $Q$ where the associated bilinear form $B_{Q}$ is symmetric. Let $\mathcal{C}=\left\{v_{1}, v_{2}, \ldots, v_{d}\right\}$ be a basis of $V$. We say that $\mathcal{C}$ is symmetric if $Q\left(v_{i}\right)=0$ and $B\left(v_{i}, v_{j}\right)=1$ for all $i, j$ with $1 \leq i<j \leq d$. In Section 3, we determine a necessary and sufficient condition for a vector space to have a symmetric basis.

Let $G$ be an extraspecial 2-group of order $2^{2 r+1}$ with $r \geq 1$, that is, $|Z(G)|=2$ and $G / Z(G) \cong \mathbb{Z}_{2}^{2 r}$. There are two extraspecial 2-groups of each order, for which we will give more details in Section 3 . Let $\bar{S}=\left\{\overline{s_{1}}, \ldots, \overline{s_{2 r}}\right\}$ be a symmetric basis of $G / Z(G)$, and for each $1 \leq i \leq 2 r$, let $s_{i}$ be a preimage of $\overline{s_{i}}$ in $G$. Notice that generally for a basis of $G / Z(G)$, the preimages of the basis elements are not necessary involutions in $G$. However in Section 3 we show that in the case where it is a symmetric basis the preimages of the basis elements are all involutions in $G$, which is crucial for the proofs of the main results. Note that $\Sigma=\operatorname{Cay}(G / Z(G), \bar{S})$ is a $2 r$-dimensional hypercube. We will prove the following result.

Theorem 1.1. Let $G$ be an extraspecial 2-group of order $2^{2 r+1}$ with $r \geq 1$ such that $G / Z(G)$ has a symmetric basis $\left\{\overline{s_{1}}, \ldots, \overline{s_{2 r}}\right\}$. Let $\Gamma=\operatorname{Cay}(G, S)$ be a Cayley graph of $G$ with $S=\left\{s_{1}, \ldots, s_{2 r}\right\}$. Then $\Gamma$ is a 2-arc-transitive cover of some $2 r$-dimensional hypercube $\Sigma$, and $\Gamma$ is a normal Cayley graph with $\operatorname{Aut}(\Gamma)=G \rtimes S_{2 r}$.

\section{Preliminaries}

Let $V$ be a $2 r$-dimensional $(r \geq 1)$ vector space over a field $\mathbb{F}_{q}$, where $q$ is a prime-power. Let $B$ be a bilinear form on $V$. We say that $B$ is symmetric if $B(u, v)=B(v, u)$ for all $u, v \in V$, and $B$ is alternating if $B(u, u)=0$ for all $u \in V$. The radical of $B$ is the subspace

$$
\operatorname{rad}(B)=\{u \in V \mid B(u, v)=0 \text { for all } v \in V\}
$$

and $B$ is said to be nondegenerate if $\operatorname{rad}(B)=\{0\}$. Let $W$ be a subspace of $V$. Define

$$
W^{\perp}=\{v \in V \mid B(w, v)=0 \text { for all } w \in W\}
$$

to be the orthogonal complement of $\mathrm{W}$. It is known that if $B$ is nondegenerate, then $\operatorname{dim}(W)+$ $\operatorname{dim}\left(W^{\perp}\right)=\operatorname{dim}(V)$. A map $Q: V \rightarrow \mathbb{F}_{q}$ is a quadratic form on $V$ if the following two conditions are satisfied: 
(i) $Q(\lambda u)=\lambda^{2} Q(u)$ for all $u \in V$ and $\lambda \in \mathbb{F}_{q}$, and

(ii) the map $B_{Q}: V \times V \rightarrow \mathbb{F}_{q}$ defined by

$$
B_{Q}(u, v)=Q(u+v)-Q(u)-Q(v)
$$

is a bilinear form.

The bilinear form $B_{Q}$ is called the associated bilinear form of $Q$. A quadratic form $Q$ is said to be nondegenerate if and only if its associated bilinear form is nondegenerate.

Let $u, v$ be two distinct vectors of $V$. We say that $\{u, v\}$ is a hyperbolic pair if $Q(u)=Q(v)=0$ and $B(u, v)=1$. By [4, Proposition 2.2.7], when $Q$ is nondegenerate and $B=B_{Q}$ is symmetric, $V$ has the following two types of standard bases, in particular, the basis is hyperbolic in case (i), and elliptic in case (ii):

(i). $\mathcal{B}=\left\{e_{1}, \ldots, e_{n / 2}, f_{1}, \ldots, f_{n / 2}\right\}$ where

$$
Q\left(e_{i}\right)=Q\left(f_{i}\right)=0, B\left(e_{i}, f_{j}\right)=\delta_{i j} \text { for all } i, j
$$

(ii). $\mathcal{B}=\left\{e_{1}, \ldots, e_{n / 2-1}, f_{1}, \ldots, f_{n / 2-1}, x, y\right\}$ where $Q\left(e_{i}\right)=Q\left(f_{i}\right)=0$,

$$
B\left(e_{i}, x\right)=B\left(e_{i}, y\right)=B\left(f_{i}, x\right)=B\left(f_{i}, y\right)=0, B\left(e_{i}, f_{j}\right)=\delta_{i j}
$$

for all $i, j, Q(x)=1, B(x, y)=1$ and $Q(y)=\zeta$ where $x^{2}+x+\zeta \in \mathbb{F}_{q}[x]$ is irreducible,

where

$$
\delta_{i j}= \begin{cases}1 & \text { if } i=j \\ 0 & \text { if } i \neq j .\end{cases}
$$

If $V$ has a hyperbolic basis, then $Q$ is said to be a hyperbolic quadratic form (or hyperbolic in short). Similarly we say that $Q$ is elliptic when $V$ has an elliptic basis.

Let $U$ be a subspace of $V$. We say that $U$ is totally singular if $Q(u)=0$ for all $u \in U$. The next result is a consequence of Witt's Lemma (see [4])

Proposition 2.1 ([5, Page 38]). Let $V$ be a d-dimensional vector space over the field $\mathbb{F}_{q}$ equipped with a nondegenerate quadratic form $Q$, and $U$ be a maximal totally singular subspace of $V$. Then

$$
\operatorname{dim} U=\frac{1}{2} \operatorname{dim} V-\delta
$$

where $\delta=0$ if $Q$ is hyperbolic, and $\delta=1$ if $Q$ is elliptic.

\section{Symmetric Basis of a Vector Space}

Let $V$ be a $2 r$-dimensional vector space over $\mathbb{F}_{2}$ with a nondegenerate quadratic form $Q: V \rightarrow \mathbb{F}_{2}$ and an associated symmetric bilinear form $B=B_{Q}$. Suppose that $\mathcal{C}=\left\{v_{1}, \ldots, v_{2 r}\right\}$ is a symmetric basis of $V$. Let $\mathcal{C}^{\prime}=\left\{c_{1}, \ldots, c_{r-1}\right\}$ where for $1 \leqslant i \leqslant r-1$, we have

$$
c_{i}=v_{2 i-1}+v_{2 i}+v_{2 i+1}+v_{2 i+2} .
$$


Let $U$ be the subspace of $V$ generated by $\mathcal{C}^{\prime}$. Then $\operatorname{dim}(U)=\frac{1}{2} \operatorname{dim}(V)-1$. Also $U$ is totally singular as $Q\left(c_{i}\right)=0$ and $B\left(c_{i}, c_{j}\right)=0$ for all $1 \leqslant i, j \leqslant r-1$. If $U$ is maximal subject to being totally singular in $V$, then $Q$ is elliptic. Otherwise $Q$ is hyperbolic, and there is a vector $v \in V \backslash U$ such that $W=\left\langle v, \mathcal{C}^{\prime}\right\rangle$ is a maximal totally singular subspace of $V$.

Let $\alpha \in V$ be a non-zero vector that is not in $\mathcal{C}^{\prime}$. We may assume that $\alpha=\mu_{1}+\mu_{2}+\cdots+\mu_{t}$, where $\mu_{i} \in \mathcal{C}$ and $\mu_{i} \neq \mu_{j}$ for all $1 \leqslant i \neq j \leqslant t$.

Lemma 3.1. $Q(\alpha)=0$ if and only if $t \equiv 0$ or $1(\bmod 4)$.

Proof. First suppose that $t=2 k$ with $k \geqslant 0$ and let $u_{i}=\mu_{2 i-1}+\mu_{2 i}$, for $i=1,2, \ldots, k$. So

$$
\begin{aligned}
& Q\left(\mu_{1}+\mu_{2}+\cdots+\mu_{2 k}\right) \\
& =Q\left(u_{1}+u_{2}+\cdots+u_{k}\right) \\
& =Q\left(u_{1}\right)+Q\left(u_{2}+\cdots+u_{k}\right)+\sum_{i=2}^{k} B\left(u_{1}, u_{i}\right) \\
& =Q\left(u_{1}\right)+Q\left(u_{2}\right)+Q\left(u_{3}+\cdots+u_{k}\right)+\sum_{i=2}^{k} B\left(u_{1}, u_{i}\right)+\sum_{i=3}^{k} B\left(u_{2}, u_{i}\right) \\
& =Q\left(u_{1}\right)+Q\left(u_{2}\right)+Q\left(u_{3}\right)+Q\left(u_{4}+\cdots+u_{k}\right)+\sum_{i=2}^{k} B\left(u_{1}, u_{i}\right)+\sum_{i=3}^{k} B\left(u_{2}, u_{i}\right)+\sum_{i=4}^{k} B\left(u_{3}, u_{i}\right) \\
& =\sum_{i=1}^{k} Q\left(u_{i}\right)+\sum_{i=1}^{k-1} \sum_{j=i+1}^{k} B\left(u_{i}, u_{j}\right) .
\end{aligned}
$$

For $1 \leqslant i<j \leqslant k$ we have

$$
\begin{aligned}
B\left(u_{i}, u_{j}\right) & =B\left(\mu_{2 i-1}+\mu_{2 i}, \mu_{2 j-1}+\mu_{2 j}\right) \\
& =B\left(\mu_{2 i-1}, \mu_{2 j-1}\right)+B\left(\mu_{2 i-1}, \mu_{2 j}\right)+B\left(\mu_{2 i}, \mu_{2 j-1}\right)+B\left(\mu_{2 i}, \mu_{2 j}\right) \\
& =0
\end{aligned}
$$

and

$$
\begin{aligned}
Q\left(u_{i}\right)= & Q\left(\mu_{2 i-1}+\mu_{2 i}\right) \\
& =Q\left(\mu_{2 i-1}\right)+Q\left(\mu_{2 i}\right)+B\left(\mu_{2 i-1}, \mu_{2 i}\right) \\
& =1 .
\end{aligned}
$$

Thus $Q(\alpha)=0$ if and only if $k \equiv 0(\bmod 2)$, that is, if and only if $t \equiv 0(\bmod 4)$.

Next suppose that $t$ is odd, and so $t=2 k+1$ with $k \geqslant 0$. Then

$$
\begin{aligned}
Q\left(\mu_{1}+\mu_{2}+\cdots+\mu_{2 k}+\mu_{2 k+1}\right) & =Q\left(\mu_{1}+\cdots+\mu_{2 k}\right)+Q\left(\mu_{2 k+1}\right)+\sum_{i=1}^{2 k} B\left(\mu_{i}, \mu_{2 k+1}\right) \\
& =Q\left(\mu_{1}+\cdots+\mu_{2 k}\right) .
\end{aligned}
$$

which implies that $Q(\alpha)=0$ if and only if $t \equiv 1(\bmod 4)$.

Before we introduce the sufficient and necessary conditions for $V$ to have a symmetric basis, we first give the following lemma. 
Lemma 3.2. Let $W$ be a nontrivial subspace of $V$ with dimension $d \equiv 2(\bmod 4)$. Suppose that $W$ has a symmetric basis and there are three pairwise perpendicular hyperbolic pairs $\{a, b\},\{c, d\},\{g, h\}$ in $W^{\perp} \backslash W$. Let

$$
W^{\prime}=W \perp\langle a, b\rangle \perp\langle c, d\rangle \perp\langle g, h\rangle .
$$

Then $W^{\prime}$ has a symmetric basis.

Proof. Let $\mathcal{C}_{W}=\left\{w_{1}, \ldots, w_{d}\right\}$ be a symmetric basis of $W$. Let

$$
\begin{aligned}
& u_{1}=a+c+d+\sum_{i=1}^{d} w_{i} ; \\
& u_{2}=b+c+d+\sum_{i=1}^{d} w_{i} ; \\
& u_{3}=c+g+h+\sum_{i=1}^{d} w_{i} ; \\
& u_{4}=d+g+h+\sum_{i=1}^{d} w_{i} ; \\
& u_{5}=g+a+b+\sum_{i=1}^{d} w_{i} ; \\
& u_{6}=h+a+b+\sum_{i=1}^{d} w_{i} .
\end{aligned}
$$

Since $d \equiv 2(\bmod 4)$, Lemma 3.1 implies $Q\left(\sum_{i=1}^{d} w_{i}\right)=1$. So $Q\left(u_{j}\right)=0$ for all $j=1,2, \ldots, 6$. For any $1 \leqslant j_{1} \neq j_{2} \leqslant 6$, one can check that

$$
B\left(u_{j_{1}}, u_{j_{2}}\right)=1
$$

Also we have $B\left(u_{j}, w_{i}\right)=1$ for all $1 \leqslant i \leqslant d$ and $j=1,2, \ldots, 6$. Therefore $\mathcal{C}_{W} \cup\left\{u_{1}, \ldots, u_{6}\right\}$ is a symmetric basis of $W^{\prime}$.

Lemma 3.3. Let $V$ be a vector space of dimension $2 r$ with nondegenerate quadratic form $Q$ such that $r \geqslant 1$.

(i). If $Q$ is hyperbolic and $r \equiv 0$ or $1(\bmod 4)$, then $V$ has a symmetric basis;

(ii). If $Q$ is elliptic and $r \equiv 2$ or $3(\bmod 4)$, then $V$ has a symmetric basis.

Proof. We prove this by using induction on $r$.

(i) Suppose that $Q$ is hyperbolic with $r \equiv 0$ or $1(\bmod 4)$. When $r=1$, a hyperbolic basis of $V$ is also a symmetric basis of $V$. Now assume the lemma holds for all $r \leqslant 4 \ell+1$ with $r \equiv 0$ or $1(\bmod 4)$, where $\ell$ is a nonnegative integer. Note that we have seen that the lemma holds when $\ell=0$. Let $r^{\prime}=4 \ell$ and suppose that $r=r^{\prime}+4=4(\ell+1)$. Let $W \leqslant V$ be a subspace of dimension $2\left(r^{\prime}+1\right)$ such that $Q$ is hyperbolic on $W$ and

$$
V=W \perp\langle a, b\rangle \perp\langle c, d\rangle \perp\langle g, h\rangle .
$$


where $\{a, b\},\{c, d\},\{g, h\}$ are hyperbolic pairs in $V \backslash W$. By induction, $W$ has a symmetric basis $\mathcal{C}_{W}=$ $\left\{w_{1}, w_{2}, \ldots, w_{2\left(r^{\prime}+1\right)}\right\}$. Take $d=2\left(r^{\prime}+1\right)$. Then $V$ has a symmetric basis $\mathcal{C}_{W} \cup\left\{u_{1}, \ldots, u_{6}\right\}$ as constructed in Lemma 3.2 .

Now suppose that $r=4(\ell+1)+1$. We may assume that

$$
V=W \perp\langle a, b\rangle \perp\langle c, d\rangle \perp\langle g, h\rangle \perp\langle x, y\rangle,
$$

where $\{a, b\},\{c, d\},\{g, h\},\{x, y\}$ are hyperbolic. Clearly $V$ contains a subspace $U$ with a symmetric basis $\mathcal{C}_{W} \cup\left\{u_{1}, \ldots, u_{6}\right\}$ as above, and let

$$
\begin{aligned}
& \alpha=x+\sum_{i=1}^{2 r^{\prime}+2} w_{i}+a+b+c+d+g+h ; \\
& \beta=y+\sum_{i=1}^{2 r^{\prime}+2} w_{i}+a+b+c+d+g+h .
\end{aligned}
$$

Then $Q(\alpha)=Q(\beta)=0$ and $B(\alpha, \beta)=1$. Also for $1 \leqslant i \leqslant 2\left(r^{\prime}+1\right)$ we have $B\left(\alpha, w_{i}\right)=B\left(\beta, w_{i}\right)=1$, and $B\left(\alpha, u_{j}\right)=B\left(\beta, u_{j}\right)=1$ for $1 \leqslant j \leqslant 6$. Hence $\mathcal{C}_{W} \cup\left\{u_{1}, \ldots, u_{6}\right\} \cup\{\alpha, \beta\}$ forms a symmetric basis of $V$.

(ii) Suppose that $Q$ is elliptic and $r \equiv 2$ or $3(\bmod 4)$. When $r=2$, let $\left\{e_{1}, f_{1}, x, y\right\}$ be an elliptic basis of $V$. Let

$$
c_{1}=e_{1}, c_{2}=f_{1}, c_{3}=x+c_{1}+c_{2}, c_{4}=y+c_{1}+c_{2}
$$

Then $Q\left(c_{i}\right)=0$ and $B\left(c_{i}, c_{j}\right)=1$ for $1 \leqslant i<j \leqslant 4$. So $\left\{c_{1}, c_{2}, c_{3}, c_{4}\right\}$ is a symmetric basis of $V$.

When $r=3$, let $\left\{e_{1}, e_{2}, f_{1}, f_{2}, x, y\right\}$ be an elliptic basis. Let $c_{1}, c_{2}, c_{3}, c_{4}$ be defined as above and let

$$
c_{5}=e_{2}+c_{1}+c_{2}+c_{3}+c_{4}, c_{6}=f_{2}+c_{1}+c_{2}+c_{3}+c_{4} .
$$

Then $\left\{c_{1}, c_{2}, c_{3}, c_{4}, c_{5}, c_{6}\right\}$ is a symmetric basis of $V$.

Now assume the lemma holds for all $r \leqslant 4 \ell+3$ with $r \equiv 2$ or $3(\bmod 4)$ where $\ell$ is a nonnegative integer. Note that we have seen that the lemma holds when $\ell=0$. Let $r^{\prime}=4 \ell+2$ and suppose that $r=r^{\prime}+4=4(\ell+1)+2$. Let $W \leqslant V$ be a subspace of dimension $2\left(r^{\prime}+1\right)$ such that $Q$ is elliptic on $W$ and

$$
V=W \perp\langle a, b\rangle \perp\langle c, d\rangle \perp\langle g, h\rangle,
$$

where $\{a, b\},\{c, d\},\{g, h\}$ are hyperbolic. By our induction $W$ has a symmetric basis

$$
\mathcal{C}_{W}=\left\{w_{1}, \ldots, w_{2 r^{\prime}+1}, w_{2 r^{\prime}+2}\right\}
$$

Then by Lemma 3.2 , when $r=4(\ell+1)+2, V$ contains a symmetric basis. When $r=4(\ell+1)+3$, let $x, y, \alpha, \beta$ be vectors of $V$ as defined in $(i)$. Then $\mathcal{C}_{W} \cup\left\{u_{1}, \ldots, u_{6}\right\} \cup\{\alpha, \beta\}$ forms a symmetric basis of $V$. 
Let $V$ be a $d$-dimensional vector space over field $\mathbb{F}_{q}$ equipped with a quadratic form $Q$ and $U$ be a $d$-dimensional vector spaces over field $\mathbb{F}_{q}$ equipped with a quadratic form $Q^{\prime}$. An isometry from $V$ to $U$ is an invertible linear map $\sigma: V \rightarrow U$ such that

$$
Q^{\prime}\left(v^{\sigma}\right)=Q(v)
$$

for all $v \in V$. Notice that (1) implies that

$$
B_{Q^{\prime}}\left(u^{\sigma}, v^{\sigma}\right)=B_{Q}(u, v)
$$

for all $u, v \in V$. If such an isometry exists, then both $U$ and $V$, and $Q$ and $Q^{\prime}$ are said to be isometric. Let $W \subseteq V$ and $W^{\prime} \subseteq U$. Then $W$ and $W^{\prime}$ are isometric if the restrictions $\left.Q\right|_{W}$ and $\left.Q^{\prime}\right|_{W^{\prime}}$ are isometric. We say that $\sigma$ is an isometry of $Q$ if $U=V$. The isometry group of $Q$ is the set of isometries of $Q$ under composition. Notice that the isometry group of $Q$ is a subgroup of the isometry group of the associated bilinear form $B_{Q}$.

Lemma 3.4. Let $V$ be a vector space of dimension $2 r$ with nondegenerate quadratic form $Q$ and a symmetric basis $\mathcal{C}=\left\{v_{1}, v_{2}, \ldots, v_{2 r}\right\}$.

(i). If $r \equiv 0$ or $1(\bmod 4)$, then $Q$ is hyperbolic;

(ii). If $r \equiv 2$ or $3(\bmod 4)$, then $Q$ is elliptic.

Proof. Let $Q_{1}$ and $Q_{2}$ be quadratic forms on $V$ such that $V$ has a symmetric basis $\mathcal{C}=\left\{c_{1}, \ldots, c_{2 r}\right\}$ with respect to $Q_{1}$ and a symmetric basis $\mathcal{C}^{\prime}=\left\{c_{1}^{\prime}, \ldots, c_{2 r}^{\prime}\right\}$ with respect to $Q_{2}$. Let $\sigma: V \rightarrow V$ be the linear map defined by

$$
\sigma: c_{i} \rightarrow c_{i}^{\prime} \text {, for } 1 \leq i \leq 2 r
$$

Then we have

$$
B_{Q_{2}}\left(c_{i}^{\sigma}, c_{j}^{\sigma}\right)=B_{Q_{1}}\left(c_{i}, c_{j}\right) \text { and } Q_{2}\left(c_{i}^{\sigma}\right)=Q_{1}\left(c_{i}\right), \text { for all } 1 \leq i, j \leq 2 r
$$

Thus $\sigma$ is an isometry of $Q_{1}$ and hence $Q_{1}$ and $Q_{2}$ have the same type. Therefore by Lemma 3.3 either $Q_{2}$ is hyperbolic with $r \equiv 0$ or $1(\bmod 4)$, or $Q_{2}$ is elliptic with $r \equiv 2$ or $3(\bmod 4)$.

Combining the results of this section, we obtain the following theorem.

Theorem 3.1. Let $V$ be a vector space of dimension $2 r$ over $\mathbb{F}_{2}$ with nondegenerate quadratic form $Q$. Then $V$ has a symmetric basis if and only if either $Q$ is hyperbolic and $r \equiv 0$ or $1(\bmod 4)$, or $Q$ is elliptic and $r \equiv 2$ or $3(\bmod 4)$.

\section{A 2-Arc-Transitive Cover of Hypercubes}

Let $G$ be an extraspecial 2-group of order $2^{2 r+1}$ with identity $\mathbf{1}(r \geqslant 1)$. Let $Z=\langle z\rangle$ be the center of $G$. Then $Z \cong \mathbb{Z}_{2}$ and $G / Z \cong \mathbb{Z}_{2}^{2 r}$ is elementary abelian. The commutator of any two elements in $G$ or the square of any element in $G$ lies in $Z$. So $G$ is a nilpotent group of class 2 . Define two functions $B: G / Z \times G / Z \rightarrow Z$ and $Q: G / Z \rightarrow Z$ as below: for any $Z x, Z y$ in $G / Z$, 


$$
\begin{gathered}
B(Z x, Z y)=[x, y] \\
Q(Z x)=x^{2} .
\end{gathered}
$$

Then $Q$ is a quadratic form on $V=G / Z$ with associated bilinear form $B$. Note that if $B(Z x, Z y)=\mathbf{1}$ for some $x, y \in G$, then $x, y$ commute. So if $B(Z x, Z y)=\mathbf{1}$ for all $y \in G$, then $Z x$ must be the identity in $G / Z$. Therefore $Q$ is nondegenerate on $G / Z$. Furthermore $B$ is symmetric as $[y, x]=[x, y]^{-1}=[x, y]$ for all $x, y \in G$.

We say that $G$ is an extraspecial 2-group of plus type if $Q$ is hyperbolic, denoted by $2_{+}^{2 r+1}$, and $G$ is an extraspecial 2-group of minus type if $Q$ is elliptic, denoted by $2_{-}^{2 r+1}$. It is known [17] that if $G=2_{+}^{2 r+1}$, then it is the central product of $r$ dihedral groups $D_{8}$, otherwise $G$ is the central product of $r-1$ dihedral groups $D_{8}$ with one quaternion group $Q_{8}$. Also Winter proved that $\operatorname{Aut}\left(2_{\epsilon}^{2 r+1}\right)=2^{2 r} . O^{\epsilon}(2 r, 2)$ where $O^{\epsilon}(2 r, 2)$ with $\epsilon \in\{+,-\}$ is an orthogonal group (see [17, Theorem 1]).

By Theorem 3.1 $G / Z$ has a symmetric basis if and only if either $G$ is of plus type with $r \equiv 0$ or $1(\bmod 4)$, or $G$ is of minus type with $r \equiv 2$ or $3(\bmod 4)$. Let $\mathcal{B}=\left\{Z g_{1}, Z g_{2}, \ldots, Z g_{2 r}\right\}$ be a symmetric basis of $G / Z$, and so for all $1 \leqslant i \neq j \leqslant 2 r$ we have that $Q\left(Z g_{i}\right)=\left(g_{i}\right)^{2}=\mathbf{1}$ and $B\left(Z g_{i}, Z g_{j}\right)=\left[g_{i}, g_{j}\right]=z$. This implies that $g_{1}, g_{2}, \ldots, g_{2 r}$ are involutions of $G$, and $g_{i} g_{j}=g_{j} g_{i} z$ for all distinct $i$ and $j$ with $1 \leqslant i, j \leqslant 2 r$. Let $\Sigma=\operatorname{Cay}(G / Z, \mathcal{B})$ and $\Gamma=\operatorname{Cay}(G, S)$ with $S=\left\{g_{1}, g_{2}, \ldots, g_{2 r}\right\}$. Thus $\Sigma$ is a $2 r$-dimensional hypercube.

To prove Theorem 1.1, we first show that $\Gamma$ is a 2 -arc-transitive cover of $\Sigma$. The Frattini subgroup $\Phi(M)$ of a group $M$ is the intersection of all maximal subgroups of $M$. If $M$ is a $p$-group, then the Frattini quotient $M / \Phi(M)$ of $M$ is isomorphic to $Z_{p}^{k}$ where $k$ is the smallest number of generators for $M$. Since $G$ is an extraspecial 2-group, we have that $\Phi(G)=Z$. Since $G / Z \cong \mathbb{Z}_{2}^{2 r}$ has a symmetric basis $\mathcal{B}$, by the Burnside Basis Theorem (see [13, Theorem 11.12]) we have that $G=\left\langle g_{1}, g_{2}, \ldots, g_{2 r}\right\rangle$.

Let $g \in G$ where $\mathbf{1} \neq g \neq z$. Then $Z g$ can be uniquely written as $Z g_{s_{1}} Z g_{s_{2}} \cdots Z g_{s_{t}}$ where $1 \leqslant s_{1}<$ $s_{2}<\cdots<s_{t} \leqslant 2 r$. So $g=z^{j} g_{s_{1}} \cdots g_{s_{t}}$ for $j=0$ or 1 .

For each $\sigma \in S_{2 r}$, define a map $\tilde{\sigma}: G \rightarrow G$ by $z^{\tilde{\sigma}}=z$, and for all $g \in G$ with $g \neq z$,

$$
g^{\tilde{\sigma}}=z^{j} g_{s_{1}^{\sigma}} \cdots g_{s_{t}^{\sigma}}
$$

where $g=z^{j} g_{s_{1}} \cdots g_{s_{t}}$ for $j=0$ or 1 . Note that $\left(Z g_{1}\right)\left(Z g_{2}\right)=Z g_{1} g_{2}=Z g_{2} g_{1}$ for any $g_{1}$ and $g_{2}$ in $G$. In particular, for all $s_{i}, s_{k}$ we have $g_{s_{i}} g_{s_{k}}=z^{j} g_{s_{k}} g_{s_{i}}$ for some $j$ and so we can deduce that $\tilde{\sigma}$ is a homomorphism. Suppose that $h \in \operatorname{Ker}(\tilde{\sigma})$ and $h \neq 1$. Since $h \neq z$, the element $h$ can be uniquely written as $z^{j} g_{s_{1}} \cdots g_{s_{t}}$ for some $s_{i} \in\{1, \ldots, 2 r\}$. Since $h^{\tilde{\sigma}}=\mathbf{1}$, we have

$$
Z=Z\left(h^{\tilde{\sigma}}\right)=Z g_{s_{1}^{\sigma}} \cdots Z g_{s_{t}^{\sigma}} .
$$

This is a contradiction as $\left\{Z g_{1}, \ldots, Z g_{2 r}\right\}$ is a basis of $G / Z$. Therefore, $\operatorname{Ker}(\tilde{\sigma})=\mathbf{1}$. Thus, $\tilde{\sigma}$ is injective and as $G$ is finite, it follows that $\tilde{\sigma}$ is surjective. Hence, $\tilde{\sigma} \in A u t(G)$.

Theorem 4.1. $S_{2 r} \leqslant \operatorname{Aut}(G)$.

Proof. Let $\phi: S_{2 r} \rightarrow \operatorname{Aut}(G)$ be the map defined as below:

$$
\phi: \sigma \rightarrow \tilde{\sigma}, \text { for each } \sigma \in S_{2 r}
$$

It is not hard to prove that $\phi$ is a homomorphism from $S_{2 r}$ into $A u t(G)$. Let $K$ be the kernel of $\phi$, that is,

$$
K=\left\{\sigma \in S_{2 r} \mid \tilde{\sigma}=\mathbf{1}\right\}
$$


Since $\tilde{\sigma}=\mathbf{1}$, we have that $g_{i}^{\tilde{\sigma}}=g_{i^{\sigma}}=g_{i}$ for all $1 \leqslant i \leqslant 2 r$. Thus $\sigma=\mathbf{1}$, and so $K=\{\mathbf{1}\}$. Therefore, $S_{2 r} \leqslant \operatorname{Aut}(G)$.

Theorem 4.2. The graph $\Gamma$ is a 2-arc-transitive Cayley graph of $G$.

Proof. Let $A=A u t(\Gamma)$. Then $S_{2 r} \leqslant A_{1}$ as $S_{2 r}$ fixes the identity element $\mathbf{1}$ of $G$. Let $N(\mathbf{1})$ be the set of neighbours of $\mathbf{1}$ in $\Gamma$. Thus $S_{2 r}$ is 2 -transitive on $N(\mathbf{1})$. Since $\Gamma$ is vertex-transitive, we have that $\Gamma$ is 2-arc-transitive.

Theorem 4.3. $\Gamma$ is a 2-arc-transitive cover for an even-dimensional hypercube.

Proof. It follows from Theorem 4.2 that $G \rtimes S_{2 r} \leqslant \operatorname{Aut}(\Gamma)$ is 2-arc-transitive on $\Gamma$. Since $|G: Z(G)| \geqslant 4$, by [11, Theorem 4.1] we have that $\Gamma$ is a cover of $\Gamma_{Z(G)} \cong \Sigma$.

Now we prove that $\Gamma$ is a normal Cayley graph for $G$. By a computation in MAgma [3], we found that $\Gamma$ is a normal Cayley graph for $G$ for all $r \in\{1,2,3\}$. Next, we show that this is true for the general case, that is, for all $r \geqslant 4$.

Let $C$ be a cycle in $\Gamma$ with $V(C)=\left\{c_{1}, \ldots, c_{t_{c}}\right\}$ and $E=\left\{\left\{c_{t_{c}}, c_{1}\right\}\right\} \cup\left\{\left\{c_{i}, c_{i+1}\right\} \mid 1 \leqslant i \leqslant t_{c}-1\right\}$ where $t_{c}$ is the length of $C$. There is a sequence $\left(s_{1}, \ldots, s_{t_{c}}\right)$ induced by $C$ where $s_{i} \in S$ for $1 \leqslant i \leqslant t_{c}$, such that $c_{1}=s_{t_{c}} c_{t_{c}}$ and $c_{i+1}=s_{i} c_{i}$ for $1 \leqslant i \leqslant t_{c}-1$. Since $s_{i}$ is an involution for all $1 \leqslant i \leqslant 2 r$, we have that

$$
s_{1} s_{2} \cdots s_{t_{c}}=\mathbf{1}
$$

and

$$
c_{i}= \begin{cases}s_{t_{c}} c_{t_{c}}, & i=1, \\ \left(s_{i-1} s_{i-2} \cdots s_{1}\right) c_{1}, & 2 \leqslant i \leqslant t_{c} .\end{cases}
$$

The sequence is uniquely determined by $C$. Since $\Gamma$ is simple, we have the following lemma.

Lemma 4.1. $s_{1} \neq s_{t_{c}}$, and $s_{i} \neq s_{i+1}$ for all $1 \leqslant i \leqslant t_{c}-1$.

We call the sequence induced by $C$ the cycle-sequence for $C$.

Lemma 4.2. Suppose that $\left(s_{1}, \ldots, s_{n}\right)$ is a sequence with $s_{i} \in S$ for all $1 \leqslant i \leqslant n$ such that $s_{1} \cdots s_{n} \in Z$. Then for each $1 \leqslant i \leqslant n$, the element $s_{i}$ appears an even number of times in the sequence.

Proof. Note that

$$
Z=Z s_{1} \cdots s_{n}=\left(Z s_{1}\right) \cdots\left(Z s_{n}\right)=\left(Z s_{m_{1}}\right)^{k_{1}} \cdots\left(Z s_{m_{t}}\right)^{k_{t}},
$$

where $\sum_{1 \leqslant i \leqslant t} k_{i}=n$ and $k_{i}$ is the number of $s_{m_{i}}$ in $\left(s_{1}, \ldots, s_{n}\right)$. Since $\left\{Z s_{1}, \ldots, Z s_{2 r}\right\}$ is a basis and $s_{i}$ is an involution for all $1 \leqslant i \leqslant 2 r$, we have that $k_{i}$ is even for all $1 \leqslant i \leqslant t$.

Let $A_{\mathbf{1}}^{[1]}$ be the automorphisms in $A_{\mathbf{1}}$ that fix each vertex in $N(\mathbf{1})$. Recall that $N(\mathbf{1})=S$. For each distinct $i$ and $j$ with $1 \leqslant i, j \leqslant 2 r$, note that the sequence $\left(s_{1}, \ldots, s_{8}\right)$ defined by

$$
s_{k}= \begin{cases}g_{i}, & \text { if } k \text { is odd }, \\ g_{j}, & \text { if } k \text { is even } .\end{cases}
$$

is an 8-cycle in $\Gamma$ which we will denote by $C_{i j}$ (see Figure 1(a)). Let $c_{1}=1$ and for each $2 \leqslant k \leqslant 8$, let $c_{k}=s_{k-1} s_{k-2} \cdots s_{1}$, in particular, we have that $c_{5}=g_{j} g_{i} g_{j} g_{i}=z$ and $c_{8}=g_{j}$. 
Let $\rho \in A_{1}^{[1]}$ and let $C_{i j}^{\rho}$ be the image of $C_{i j}$ under $\rho$ with $V\left(C_{i j}^{\rho}\right)=\left\{u_{k} \mid u_{k}=c_{k}^{\rho}, 1 \leqslant k \leqslant 8\right\}$. Since $g_{i}, g_{j} \in N(1)$ and $\rho \in A_{1}^{[1]}$, we have that $u_{1}=\mathbf{1}, u_{2}=g_{i}$ and $u_{8}=g_{j}$. Let $\left\{a_{1}, \ldots, a_{8}\right\}$ be the cycle-sequence for $C_{i j}^{\rho}$, and so $a_{1}=g_{i}$ and $a_{8}=g_{j}$ (see Figure $1(\mathrm{~b})$ ).

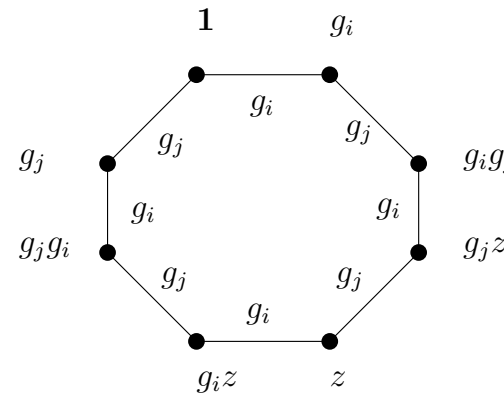

(a)

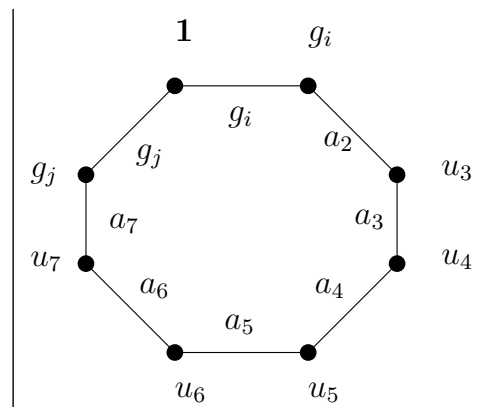

(b)

Figure 1: The 8-cycle $C_{i j}$ and its image under $\rho \in A_{1}^{[1]}(1 \leqslant i, j \leqslant 2 r, i \neq j)$

Lemma 4.3. Suppose that $z^{\rho} \neq z$. Then for $1 \leqslant i \leqslant 8$, the element $a_{i}$ appears exactly twice in the cycle sequence $\left(s_{1}, \ldots, s_{8}\right)$. Moreover, $a_{1}, a_{2}, a_{3}, a_{4}$ are pairwise distinct, and $a_{5}, a_{6}, a_{7}, a_{8}$ are pairwise distinct.

Proof. By Lemma 4.1 we have that $a_{1} \neq a_{8}$ and $a_{i} \neq a_{i+1}$ for all $1 \leqslant i \leqslant 7$. Suppose that $a_{3}=a_{1}$. Then by (3), we have that

$$
\begin{aligned}
u_{5} & =a_{4} a_{3} a_{2} a_{1} \\
& =a_{4} a_{1} a_{2} a_{1} \\
& =a_{4} a_{2} z \\
& =a_{2} a_{4} .
\end{aligned}
$$

If $a_{2}=a_{4}$, then $u_{5}=\mathbf{1}$, which is a contradiction. Thus $a_{2} \neq a_{4}$, which implies that there is a 2-path in $\Gamma$ connecting 1 and $u_{5}$. Since $\rho \in A$ and $z^{\rho}=u_{5}$, we have that there is a 2-path in $\Gamma$ connecting 1 and $z$, which leads to a contradiction. Hence $a_{3} \neq a_{1}$. If $a_{4}=a_{1}$, then

$$
\begin{aligned}
u_{5} & =a_{4} a_{3} a_{2} a_{1} \\
& =a_{1} a_{3} a_{2} a_{1} \\
& =a_{3} a_{2},
\end{aligned}
$$

and by the same arguments, we deduce that $a_{4} \neq a_{1}$. Suppose that $a_{2}=a_{4}$. Then $u_{5}=a_{4} a_{3} a_{2} a_{1}=$ $a_{1} a_{3}$, which leads to a contradiction by the same arguments. Thus $a_{1}, a_{2}, a_{3}, a_{4}$ are pairwise distinct. By (3) we have that

$$
u_{8}=a_{7} a_{6} a_{5} u_{5} .
$$

Since $\mathbf{1}=u_{1}=a_{8} u_{8}$, we have that $\mathbf{1}=a_{8} a_{7} a_{6} a_{5} u_{5}$. Since $S$ consists of involutions, we have that $u_{5}=a_{5} a_{6} a_{7} a_{8}$. Then by the same arguments, we may conclude that $a_{5}, a_{6}, a_{7}, a_{8}$ are pairwise distinct. Therefore, by Lemma 4.2 , we conclude that each term in $\left\{a_{1}, \ldots, a_{8}\right\}$ appears exactly twice.

Lemma 4.4. The cycle $C_{i j}$ is fixed pointwise by $\rho$. 
Proof. We first show that $z^{\rho}=z$. Suppose to the contrary that $z^{\rho} \neq z$. Since $u_{5}=z^{\rho}$, we have that $\left(N(z) \cap C_{i j}\right)^{\rho}=\left\{g_{i} z, g_{j} z\right\}^{\rho}=\left\{u_{4}, u_{6}\right\}$. Recall that $u_{4}=a_{3} a_{2} a_{1}$ and $u_{6}=a_{5} a_{4} a_{3} a_{2} a_{1}$.

Suppose that $\left(g_{i} z\right)^{\rho}=u_{4}$. Let $g_{k} \in S$ be such that $g_{k} \neq a_{i}$ for all $1 \leqslant i \leqslant 3$, and let $C_{i k}$ be an 8-cycle. Note that $C_{i k}$ has the same shape as in Figure 1(a). Thus $g_{i} z$ and $g_{k}$ are connected by a 2-path in $C_{i k}$, and so we have that $\left(g_{i} z\right)^{\rho}$ and $g_{k}^{\rho}$ are connected by a 2-path, that is, $u_{4}$ and $g_{k}$ are connected by a 2-path in $\Gamma$. We may assume that $x_{2} x_{1} u_{4}=g_{k}$ for some $x_{1}, x_{2} \in S$ where $x_{1} \neq x_{2}$, and so $x_{2} x_{1} a_{3} a_{2} a_{1} g_{k}=1$. By Lemma 4.2 and Lemma 4.3 , we must have that $g_{k}=a_{i}$ for some $1 \leqslant i \leqslant 3$, which leads to a contradiction. Thus $\left(g_{i} z\right)^{\rho} \neq u_{4}$, and so $\left(g_{i} z\right)^{\rho}=u_{6}$.

By Lemma 4.3, $a_{5}=a_{j}$ for some $1 \leqslant j \leqslant 3$. Suppose that $a_{5}=a_{1}$. Then $\left(g_{i} z\right)^{\rho}=u_{6}=$ $a_{1} a_{4} a_{3} a_{2} a_{1}=a_{4} a_{3} a_{2} z$. Let $g_{k^{\prime}} \in S$ such that $g_{k^{\prime}} \neq a_{i}$ for $2 \leqslant i \leqslant 4$, and let $C_{i k^{\prime}}$ be the corresponding 8-cycle. Since $g_{i} z$ and $g_{k^{\prime}}$ are joined by a 2-path, we have that $u_{6}$ and $g_{k^{\prime}}$ are joined by a 2-path, and so we have $y_{2} y_{1} a_{4} a_{3} a_{2} g_{k^{\prime}}=z$ for some $y_{1}, y_{2} \in S$ where $y_{1} \neq y_{2}$. Then by similar arguments, we conclude that $g_{k^{\prime}}=a_{j}$ for some $2 \leqslant j \leqslant 4$, which is a contradiction. For the remaining two cases where $a_{5}=a_{2}$ or $a_{5}=a_{3}$, we can obtain contradictions following similar arguments. Hence $\left(g_{i} z\right)^{\rho} \neq u_{6}$, which leads to a contradiction to the fact that $\left(g_{i} z\right)^{\rho} \in\left\{u_{4}, u_{6}\right\}$. Therefore, $z^{\rho}=z$, that is, $u_{5}=z$.

Recall that $u_{5}=a_{4} a_{3} a_{2} a_{1}=a_{5} a_{6} a_{7} a_{8}$ where $a_{1}=g_{i}$ and $a_{8}=g_{j}$. By Lemma 4.2 we have that $a_{1}=a_{3}=g_{i}, a_{2}=a_{4}, a_{5}=a_{7}$ and $a_{6}=a_{8}=g_{j}$ as $u_{5}=z$ and $a_{i} \neq a_{i+1}$ for all $1 \leqslant i \leqslant 7$. If $a_{2}=a_{4}=g_{j}$ and $a_{5}=a_{7}=g_{i}$, then $C_{i j}$ and $C_{i j}^{\rho}$ have the same cycle sequence, that is, $C_{i j}^{\rho}=C_{i j}$.

Suppose to the contrary that $a_{2}=a_{4} \neq g_{j}$. Since $u_{5}=z$, we have that $u_{4}=a_{4} z=a_{2} z$ and $u_{6}=a_{5} z$. Recall that $\left\{g_{i} z, g_{j} z\right\}^{\rho}=\left\{u_{4}, u_{6}\right\}$. Since $a_{2} \in S$, we may assume that there exists $1 \leqslant t \leqslant 2 r$ such that $a_{2}=g_{t}$ with $t \neq i$ and $t \neq j$. Suppose that $\left(g_{i} z\right)^{\rho}=u_{4}=a_{2} z$, that is, $\left(g_{i} z\right)^{\rho}=g_{t} z$. Let $C_{i t}$ be the corresponding 8-cycle. Thus $g_{t}$ and $g_{i} z$ are connected by a 2-path in $C_{i t}$, and so we have that $g_{t}$ and $\left(g_{i} z\right)^{\rho}$ are connected by a 2-path. Thus there exists $w_{1}, w_{2} \in S$ such that $w_{1} \neq w_{2}$ and $w_{2} w_{1} g_{t}=g_{t} z$, that is, $w_{1} w_{2}=z$, which is a contradiction as $Z w_{1}$ and $Z w_{2}$ are base elements. Hence $\left(g_{i} z\right)^{\rho}=u_{6}=a_{5} z$, that is, $\left(g_{j} z\right)^{\rho}=u_{4}=a_{2} z$.

Recall that $a_{2}=g_{t}$ where $t \neq i$ and $t \neq j$. Let $C_{j t}$ be the corresponding 8-cycle. Note that $C_{j t}$ has the same shape as in Figure 1(a). Thus $g_{j} z$ and $g_{t}$ are connected by a 2-path. Hence $\left(g_{j} z\right)^{\rho}=a_{2} z=g_{t} z$ and $g_{t}$ are connected by a 2-path. Suppose that there exist $u_{1}, u_{2} \in S$ such that $u_{2} u_{1} g_{t}=g_{t} z$. Thus we have that $u_{1} u_{2}=z$, which is a contradiction. Hence $a_{2}=a_{4}=g_{j}$.

By a similar argument, we may conclude that $a_{5}=a_{7}=g_{i}$. Therefore $C_{i j}^{\rho}=C_{i j}$.

Corollary 4.1. Let $v \in N(\mathbf{1})$. Then $\rho \in A_{v}^{[1]}$.

Proof. Let $w \in N(v)$. We may assume that $v=g_{1}$, and so $w=g_{1} g_{i}$ for some $1 \leqslant i \leqslant 2 r$. If $i=1$, then $w=1$, and so $\rho$ fixes 1. Suppose that $i \neq 1$, and consider the 8-cycle $C_{1 i}$. By Lemma 4.4. $C_{1 i}^{\rho}=C_{1 i}$, that is, $\rho$ fixes each vertex on $C_{1 i}$, and so $w^{\rho}=w$. Therefore $\rho \in A_{v}^{[1]}$.

Lemma 4.5. $A_{1}^{[1]}=\{1\}$.

Proof. By Corollary 4.1 we have that $A_{1}^{[1]} \leqslant A_{v}^{[1]}$. Since $\Gamma$ is vertex-transitive and finite, we have that $\left|A_{1}^{[1]}\right|=\left|A_{v}^{[1]}\right|$, which implies that $A_{1}^{[1]}=A_{v}^{[1]}$. Since $\Gamma$ is vertex-transitive, it follows that $A_{u}^{[1]}=A_{w}^{[1]}$ for each $u \in V(\Gamma)$ and $w \in N(u)$. Thus by connectivity $A_{\mathbf{1}}^{[1]}=A_{u}^{[1]}$ for all $u \in V(\Gamma)$, and so $A_{\mathbf{1}}^{[1]}=\{\mathbf{1}\}$.

Proof of Theorem 1.1. By Lemma 4.5 we have $A_{\mathbf{1}} \cong A_{\mathbf{1}}^{N(\mathbf{1})}=S_{2 r}$. Further by Theorem 4.1 we obtain $A_{\mathbf{1}}=\operatorname{Aut}(G, S)$. Therefore $A=G \rtimes S_{2 r}$, namely, $\Gamma$ is a normal Cayley graph for $G$. Also in 
Theorem 4.3 we have proved that $\Gamma$ is a 2 -arc-transitive cover of a hypercube of dimension $2 r$. This completes the proof of Theorem 1.1 .

\section{Acknowledgements}

This research is part of my Ph.D project at the Centre for the Mathematics of Symmetry and Computation of the University of Western Australia. I would like to express my gratitude to my supervisors Dr. Michael Giudici and Dr. Cai Heng Li, who offered continuous advice and encouragement throughout the progress of this paper.

I would also like to thank the referees of this paper for their great suggestions, which helped me a lot in improving the presentation of this paper.

\section{References}

[1] Alspach, B., Conder, M. D., Marušıč, D., And Xu, M.-Y. A classification of 2-arc-transitive circulants. Journal of Algebraic Combinatorics 5, 2 (1996), 83-86.

[2] BabAi, L. Arc transitive covering digraphs and their eigenvalues. Journal of Graph Theory 9, 3 (1985), 363-370.

[3] Bosma, W., Cannon, J., And Playoust, C. The Magma algebra system I: The user language. Journal of Symbolic Computation 24, 3-4 (1997), 235-265.

[4] Burness, T. C., And Giudici, M. Classical Groups, Derangements And Primes, vol. 25. Cambridge University Press, 2016.

[5] Burness, T. C., And Giudici, M. Classical groups, derangements and primes, vol. 25 of Australian Mathematical Society Lecture Series. Cambridge University Press, Cambridge, 2016.

[6] Du, S.-F., Kwak, J. H., And Xu, M.-Y. 2-Arc-transitive regular covers of complete graphs having the covering transformation group $Z_{p}^{3}$. Journal of Combinatorial Theory, Series B 93, 1 (2005), 73-93.

[7] Du, S.-F., Malnič, A., And Marušič, D. Classification of 2-arc-transitive dihedrants. Journal of Combinatorial Theory, Series B 98, 6 (2008), 1349-1372.

[8] Du, S.-F., Marušič, D., And Waller, A. O. On 2-arc-transitive covers of complete graphs. Journal of Combinatorial Theory. Series B 74, 2 (1998), 276-290.

[9] Ivanov, A. A., And Praeger, C. E. On finite affine 2-arc transitive graphs. European Journal of Combinatorics 14, 5 (1993), 421-444.

[10] Praeger, C. E. Bipartite 2-arc-transitive graphs. Australas. J. Combin 7 (1993), 21-36.

[11] Praeger, C. E. An O'Nan-Scott theorem for finite quasiprimitive permutation groups and an application to 2-arc transitive graphs. Journal of the London Mathematical Society 2, 2 (1993), $227-239$. 
[12] Praeger, C. E. Finite transitive permutation groups and bipartite vertex-transitive graphs. Illinois Journal of Mathematics 47, 1-2 (2003), 461-475. Special issue in honor of Reinhold Baer (1902-1979).

[13] Rose, J. S. A course on group theory. Courier Corporation, 1994.

[14] Sabidussi, G. On a class of fixed-point-free graphs. Proceedings of the American Mathematical Society 9, 5 (1958), 800-804.

[15] SpIgA, P. Enumerating groups acting regularly on a $d$-dimensional cube. Comm. Algebra 37, 7 (2009), 2540-2545.

[16] Weiss, R. The nonexistence of 8-transitive graphs. Combinatorica 1, 3 (1981), 309-311.

[17] Winter, D. L. The automorphism group of an extraspecial p-group. Rocky Mountain J. Math. 2, 2 (1972), 159-168.

[18] XU, M.-Y. Automorphism groups and isomorphisms of Cayley digraphs. Discrete Mathematics 182, 1 (1998), 309-319. 